\title{
The Genome Sequence of Five Highly Pathogenic Isolates of Fusarium oxysporum f. sp. lini
}

\author{
Alexander Kanapin,, ${ }^{1,2}$ Anastasia Samsonova, ${ }^{1,2}$ Tatyana Rozhmina, ${ }^{3}$ Michael Bankin, ${ }^{1}$ \\ Anton Logachev, ${ }^{2}$ and Maria Samsonova ${ }^{1, \dagger}$ \\ ${ }^{1}$ Institute for Applied Mathematics and Mechanics, Peter the Great St. Petersburg Polytechnic University, St. Petersburg, \\ 195251 Russian Federation \\ ${ }^{2}$ Centre for Genome Bioinformatics, St. Petersburg State University, St. Petersburg, 199034 Russian Federation \\ ${ }^{3}$ Federal Research Center for Bast Fiber Crops, Torzhok 172002, Russia
}

Accepted 19 June 2020.

\begin{abstract}
Fusarium wilt is the most destructive fungal disease in flax, limiting flax cultivation in all the main flax and linseed growing countries. The causative agent is seedborne and soilborne fungus $F$. oxysporum f. sp. lini. Here, we report, for the first time, genome assemblies of five highly pathogenic isolates of Fusarium oxysporum f. sp. lini, namely monoisolate 39 and strains F329, F324, F282, F287. In addition, syntenic analysis provided a powerful approach to distinguish between core and lineage-specific parts of the genome. These results lay a solid foundation for comparative genomics studies of plant fungal pathogens, evolution of pathogenicity, and virulence factors underlying the dynamics of host-pathogen interactions, thus eventually offering solutions to Fusarium disease control.
\end{abstract}

Keywords: flax, Fusarium oxysporum, Fusarium wilt, genome

Fusarium oxysporum is a genetically diverse species complex of different formae speciales (f. sp.) found to exhibit narrow host adaptation and to exploit a broad range of environmental niches (Zhang and Ma 2017). Fusarium oxysporum formae speciales include both nonpathogenic and pathogenic strains capable of causing severe disease in plants, endangering production of economically important crops (Johnson et al. 1998).

Pathogenic fungal species genomes, with Fusarium oxysporum presenting no exception to the rule, are typically divided into two functionally distinct core and lineage-specific (LS) parts (Armitage et al. 2018; Han et al. 2001; Ma et al. 2010; Zhang and Ma 2017).

The core part genes, essential for key cellular processes and metabolic pathways, are highly conserved across Fusarium

Genome data has been deposited in the National Center for Biotechnology Information GenBank database, BioProject accession number PRJNA630722.

${ }^{\dagger}$ Corresponding author: M. Samsonova; m.g.samsonova@gmail.com

Funding: This work was supported by the Russian Science Foundation, grant 19-16-00030 to A. Kanapin, T. Rozhmina, M. Bankin, and M. Samsonova (genome assemblies). A. Samsonova and A. Logachev were supported by Saint Petersburg State University (project reference 51148284) (synteny analysis).

The author(s) declare no conflict of interest.

(c) 2020 The American Phytopathological Society species. In contrast with the core, LS regions are highly dynamic and often gene-sparse. Specifically, they are enriched in transposable elements and repeats as well as various pathogenicity-associated proteins (e.g., secreted proteins and proteins involved in secondary metabolism) (Schmidt et al. 2013). The LS chromosome content defines the formae speciales host range and, when transferred to a nonpathogenic strain, can confer pathogenicity on a new host (Ma et al. 2010).

In recent years, many genomes of Fusarium oxysporum have been sequenced (Armitage et al. 2018; García-Bastidas et al. 2014; Ma et al. 2010; Schmidt et al. 2016; Seo et al. 2020; Williams et al. 2016). By far, the most accurately sequenced F. oxysporum genome is Fusarium oxysporum f. sp. lycopersici isolate 4287, assembled from whole-genome shotgun fragments, with subsequent anchoring of the assemblies to an optical map (Ma et al. 2010). The LS region of isolate 4287 includes the ends of chromosomes 1 and 2 as well as whole chromosomes 3, 6, 14, and 15 .

Fusarium oxysporum f. sp. lini, being one the most dangerous fungal pathogens in flax, is a causative agent of Fusarium wilt. It invades the plant through roots and spreads inside the vascular bundle. After germination, it develops microconidia, blocking water and nutrient flow, which leads to plant wilt, yellowing of lower parts, and death (Michielse and Rep 2009; Olivain et al. 2003; Rozhmina 2002; Rozhmina and Loshakova 2016). The fungus produces mycotoxins and enzymes hydrolyzing cell-wall components (cellulases, pectinases, glucuronidases, and others) that facilitate host tissue penetration.

Most domesticated flax varieties are either highly or moderately resistant to Fusarium wilt. However, a rapid decrease of genetic diversity of flax cultivars and the never-ending hostpathogen arms race contribute to a substantially increased risk of developing disease. Furthermore, the climate change may result in increased aggressiveness of individual pathogen races and the loss of resistance by flax varieties.

Given the economic importance of flax cultivation, the above calls for swift action, including comprehensive characterization of the pathogen population at the genomic level, thus speeding the engineering of fiber and oil flax varieties with effective pathogen resistance. Here, we report genome assemblies of five different highly pathogenic Fusarium oxysporum f. sp. lini isolates, i.e., the 39 monoisolate and four strains, F329, F324, F282, and F287.

Unlike the 39 monoisolate, for which a DNA sample was obtained from a single spore culture, the cultures of the other 
Table 1. Genome assembly statistics of Fusarium oxysporum f. sp. lini isolates

\begin{tabular}{|c|c|c|c|c|c|}
\hline \multirow[b]{2}{*}{ Statistics } & \multicolumn{5}{|c|}{ Isolate } \\
\hline & 39 & F282 & F287 & F324 & F329 \\
\hline Sequencing technology & PacBio, Illumina & Illumina & Illumina & Illumina & Illumina \\
\hline Coverage & $100+200$ & 60 & 60 & 60 & 60 \\
\hline Number of scaffolds & 26 & 185 & 180 & 35 & 197 \\
\hline Genome size (bp) & $69,459,321$ & $47,954,500$ & $47,606,230$ & $47,502,719$ & $46,883,008$ \\
\hline GC content $(\%)$ & 48.08 & 48.51 & 48.54 & 48.49 & 48.52 \\
\hline $\mathrm{N}_{50}(\mathrm{bp}), \mathrm{RaGOO}(\mathrm{ABYSS})$ & $5,280,230$ & $4,511,164(66,518)$ & $4,538,424(65,699)$ & $4,460,199(45,159)$ & $4,561,878(54,894)$ \\
\hline $\mathrm{L}_{50}$ & 5 & 5 & 5 & 5 & 5 \\
\hline Longest contig (bp) & $9,704,848$ & $6,215,061$ & $5,987,481$ & $6,558,844$ & $6,163,813$ \\
\hline Contigs $>50 \mathrm{~kb}$ & 20 & 20 & 18 & 18 & 18 \\
\hline BUSCO completeness $(\%)$ & 99.8 & 99.3 & 99.2 & 99.2 & 99.2 \\
\hline Number of predicted genes & 14,233 & 13,846 & 13,822 & 13,883 & 13,836 \\
\hline Repetitive sequences (bp) & $3,199,822$ & 614,392 & 594,345 & 606,246 & 553,256 \\
\hline NCBI accession & JABJUA000000000 & JABJUB000000000 & JABJUC000000000 & JABJUD000000000 & JABJUE000000000 \\
\hline BioSample & SAMN14843692 & SAMN14843945 & SAMN14843946 & SAMN14843947 & SAMN14843948 \\
\hline
\end{tabular}

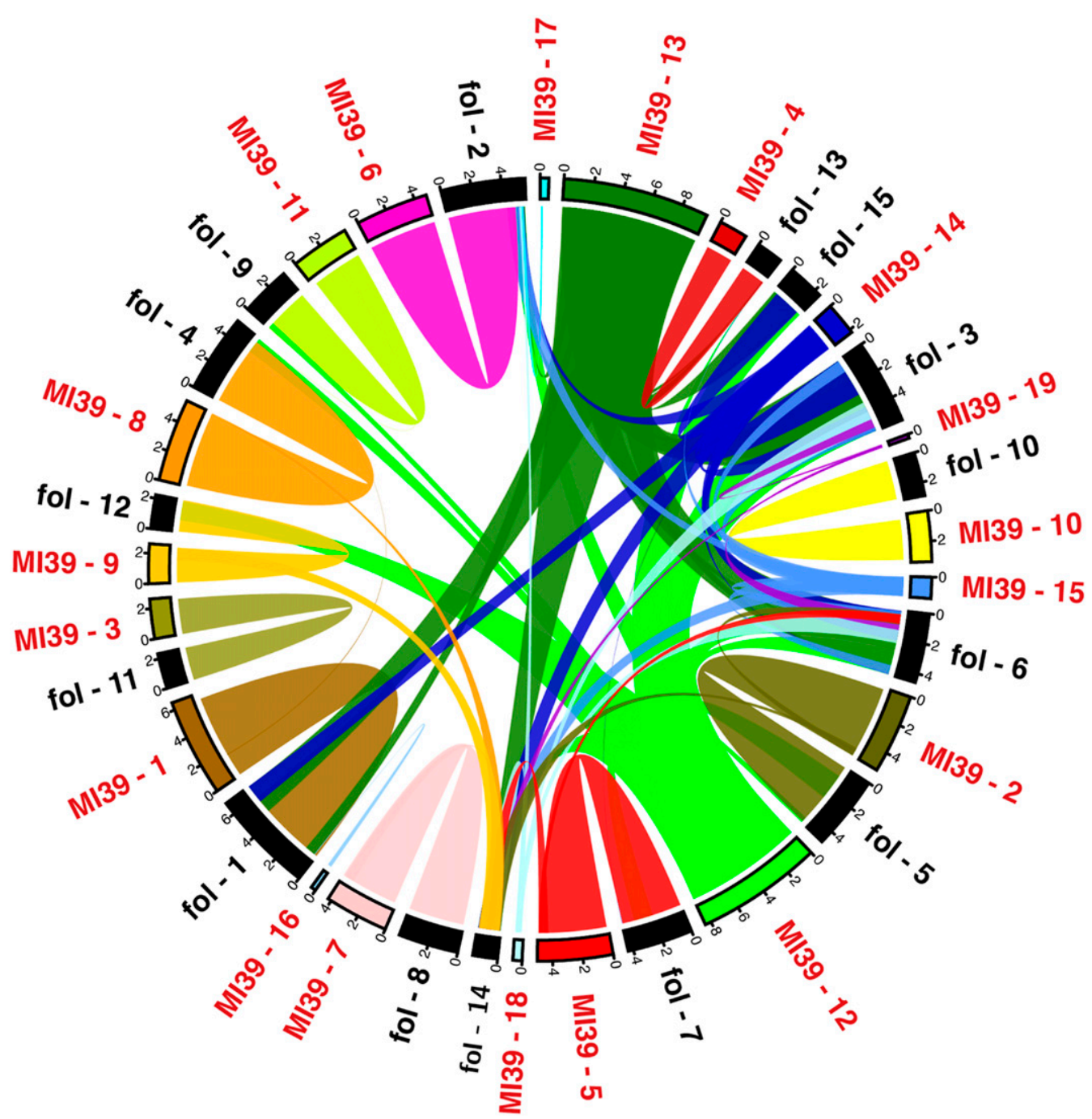

Fig. 1. Synteny of chromosomes between the Fusarium oxysporum f. sp. lycopersici genome and the MI39 monoisolate of $F$. oxysporum f. sp. lini genome assembly. Relationships are shown through linking homologous genome regions bundled with Circos software (v. 0.69-9) (Krzywinski et al. 2009). MI39 chromosome 20 is not shown, as it presents no significant homology with F. oxysporum f. sp. lycopersici. Fusarium oxysporum f. sp. lycopersici chromosomes (fol) are shown in black, while colored chromosomes correspond to the 39 monoisolate (MI39). Numbers on the chromosome scales correspond to length in megabases. 
pathogenic strains were grown from mixed spores belonging to the same strain. All cultures were grown in liquid Chapek medium and DNA was extracted with NucleoSpin Plant II (Macherey-Nagel), with the addition of RNAse A. Monoisolate 39 was sequenced to $100 \times$ coverage using the PacBio RS II system at the Center of Genome Research and Biocomputing of Oregon State University and to 200x coverage with Illumina HighSeq (150 bp, paired-end reads) (Novogene). The remaining four samples, namely, F329, F324, F282, F287, were sequenced to $60 \times$ coverage each (150 bp, paired-end reads) using Illumina HighSeq (Novogene).

The MI39 genome, as the only one sequenced with highcoverage long reads, was used for a reference assembly. The initial assembly was performed with Canu package (v. 1.4) using the default settings (Koren et al. 2017). Further polishing and correction of the assembly was made by Pilon software version 1.23 (Walker et al. 2014), using sequencing reads generated by Illumina HighSeq with one round of polishing. The error rate $(5.4 \mathrm{e}-5)$ for the assembly was estimated as a fraction of ambiguous or low-quality positions in the polished assembly, as reported by Pilon. Finally, we used the RaGOO package (v. 1.1) (Alonge et al. 2019) to build a reference-guided assembly of chromosomes and supercontigs. The Fusarium oxysporum f. sp. lycopersici isolate 4287 genome (EnsEMBL Fungi database v.46) was used as a reference for the assembly.

Table 2. Overlap between core parts of MI39 assembly and Fusarium oxysporum f. sp. lycopersici genome

\begin{tabular}{lcc}
\hline $\begin{array}{l}\text { MI39 assembly } \\
\text { chromosome }\end{array}$ & $\begin{array}{c}\text { F. oxysporum f. sp. lycopersici } \\
\text { chromosome }\end{array}$ & Overlap \\
\hline 1 & 1 & 0.87905474 \\
2 & 5 & 0.87243908 \\
3 & 11 & 0.83080631 \\
4 & 13 & 0.87938439 \\
5 & 7 & 0.83045263 \\
6 & 2 & 0.95364461 \\
7 & 8 & 0.9029552 \\
8 & 4 & 0.8572433 \\
9 & 12 & 0.82752456 \\
10 & 10 & 0.78257647 \\
11 & 9 & 0.79336214 \\
\hline
\end{tabular}

Table 3. Overlap between MI39 genome and genomes of four isolates

\begin{tabular}{lccccc}
\hline & & \multicolumn{4}{c}{ Isolate } \\
\cline { 3 - 6 } Chromosome & Length (bp) $)^{\mathbf{a}}$ & $\mathbf{F 2 8 2}$ & $\mathbf{F 2 8 7}$ & $\mathbf{F 3 2 4}$ & F329 \\
\hline 1 & $6,499,767$ & 0.99 & 0.99 & 0.99 & 0.99 \\
2 & $5,280,230$ & 0.99 & 0.99 & 0.99 & 0.99 \\
3 & $2,635,713$ & 0.99 & 0.99 & 0.99 & 0.99 \\
4 & $1,897,814$ & 0.99 & 0.99 & 0.99 & 0.99 \\
5 & $4,998,367$ & 0.99 & 0.99 & 0.99 & 0.99 \\
6 & $4,823,064$ & 0.99 & 0.99 & 0.99 & 0.99 \\
7 & $4,235,930$ & 0.99 & 0.99 & 0.99 & 0.99 \\
8 & $5,505,808$ & 0.99 & 0.99 & 0.99 & 0.99 \\
9 & $2,616,529$ & 0.99 & 0.99 & 0.99 & 0.99 \\
10 & $3,368,021$ & 0.99 & 0.99 & 0.99 & 0.98 \\
11 & $3,904,028$ & 0.99 & 0.99 & 0.99 & 0.99 \\
12 & $8,406,827$ & 0.84 & 0.79 & 0.86 & 0.80 \\
13 & $9,704,848$ & 0.78 & 0.79 & 0.83 & 0.76 \\
14 & $2,215,301$ & 0.78 & 0.75 & 0.85 & 0.78 \\
15 & $1,413,315$ & 0.81 & 0.74 & 0.83 & 0.78 \\
16 & 330,890 & 0.99 & 0.99 & 0.99 & 1 \\
17 & 562,849 & 0.99 & 0.99 & 0.99 & 0.99 \\
18 & 610,545 & 0.96 & 0.98 & 0.97 & 0.98 \\
19 & 276,224 & 0.94 & 0.97 & 0.92 & 0.97 \\
20 & 34,875 & 0.99 & 0.99 & 0.84 & - \\
Genome & & 0.97 & 0.97 & 0.98 & 0.96 \\
\hline & & & & \\
& & & & &
\end{tabular}

${ }^{a}$ Length of the MI39 assembly chromosome in base pairs.
The resulting MI39 reference genome assembly consists of 29 scaffolds, of which 20 were defined as chromosomes, based on i) their homology to the reference Fusarium oxysporum f. sp. lycopersici genome and ii) similarity to the genome assemblies of the other isolates. The remaining nine scaffolds are listed as separate entities.

The genomes of the remaining four isolates, namely, F282, F287, F324, and F329, were constructed using abyss-pe assembler from the ABYSS package (v. 2.1.5) (Simpson et al. 2009). The resulting contigs were assembled into scaffolds with RaGOO, using the MI39 genome assembly as a reference. Importantly, the reference-guided genome assemblies were highly efficient yielding MI39 chromosomes, encompassing the total of approximately $90 \%$ of the short contigs.

Longer supercontigs $(>2 \mathrm{kbp}$ ) failing to map onto MI39 chromosomes are included into submission as separate entities.

The repeated regions were masked with RepeatMasker. The assemblies based on Illumina-only sequenced data demonstrate relatively low fraction of repetitive sequences in comparison with the MI39 reference genome. Since short-read genome assemblers usually generate low-quality contigs from highly repetitive short reads, it is unsurprising to observe relatively low number of repetitive regions in the respective assemblies. The quality of all assemblies was assessed using QUAST (v 5.0.2) (Gurevich et al. 2013). We used BUSCO (v 4.0.4) (Seppey et al. 2019) with "hypocreales_odb10" as -1 parameter for gene prediction and functional annotation of the proteins. The statistics and quality-control results for the five newly assembled genomes is shown in Table 1. For the assemblies generated from Illumina reads only, $\mathrm{N}_{50}$ values for both RaGOO and ABYSS are reported.

While the core part of the $F$. oxysporum genomes is highly conserved between various formae speciales, the LS part is highly variable. Importantly, it contains the vast majority of genes responsible for host-specific pathogens. We used the Satsuma2 package (Grabherr et al. 2010) to reveal complex syntenic relationships between the MI39 reference genome assembly and the F. oxysporum f. sp. lycopersici genome and to identify the core part of the genome (Fig. 1). The assembled chromosomes 1 through 11 of MI39 demonstrate a high degree of overall similarity $(>75 \%)$ with $F$. oxysporum f. sp. lycopersici chromosomes 1, 2, 4, 5, and 7 through 13 (Table 2), which, according to Ma et al. (2010), compose the core part of F. oxysporum f. sp. lycopersici genome.

In-depth analyses of synteny between the MI39 genome and the remaining four assemblies of $F$. oxysporum $\mathrm{f}$. sp. lini show an extremely high level of sequence overlap ( $>98 \%)$, calculated as a ratio of total length of syntenic regions to a given chromosome length for all five genomes observed for chromosomes 1 through 11 and 16 through 19 (Table 3). We therefore attribute these chromosomes to the core part of $F$. oxysporum $\mathrm{f}$. sp. lini. genome, while the rest of the MI39 reference assembly chromosomes (namely, chromosomes 12 through 15 and 20 [Table 3]) were attributed to the LS part.

The genome data has been deposited in the National Center for Biotechnology Information GenBank database, BioProject accession number PRJNA630722. Individual accessions and BioSample IDs for each assembly are presented in Table 1.

\section{LITERATURE CITED}

Alonge, M., Soyk, S., Ramakrishnan, S., Wang, X., Goodwin, S., Sedlazeck, F. J., Lippman, Z. B., and Schatz, M. C. 2019. RaGOO: Fast and accurate reference-guided scaffolding of draft genomes. Genome Biol. 20:224.

Armitage, A. D., Taylor, A., Sobczyk, M. K., Baxter, L., Greenfield, B. P. J., Bates, H. J., Wilson, F., Jackson, A. C., Ott, S., Harrison, R. J., and 
Clarkson, J. P. 2018. Characterisation of pathogen-specific regions and novel effector candidates in Fusarium oxysporum f. sp. cepae. Sci. Rep. $8: 13530$.

García-Bastidas, F., Ordóñez, N., Konkol, J., Al-Qasim, M., Naser, Z., Abdelwali, M., Salem, N., Waalwijk, C., Ploetz, R. C., and Kema, G. H. J. 2014. First report of Fusarium oxysporum f. sp. cubense tropical race 4 associated with panama disease of banana outside Southeast Asia. Plant Dis. 98:694.

Grabherr, M. G., Russell, P., Meyer, M., Mauceli, E., Alföldi, J., Di Palma, F., and Lindblad-Toh, K. 2010. Genome-wide synteny through highly sensitive sequence alignment: Satsuma. Bioinformatics 26:1145-1151.

Gurevich, A., Saveliev, V., Vyahhi, N., and Tesler, G. 2013. QUAST: Quality assessment tool for genome assemblies. Bioinformatics 29: 1072-1075.

Han, Y., Liu, X., Benny, U., Kistler, H. C., and VanEtten, H. D. 2001. Genes determining pathogenicity to pea are clustered on a supernumerary chromosome in the fungal plant pathogen Nectria haematococca. Plant J. 25:305-314.

Johnson, D. D., Flaskerud, G. K., Taylor, R. D., and Satyanarayana, V. 1998. Economic impacts of Fusarium head blight in wheat. Agricultural economics report 396. North Dakota State University, Fargo, ND, U.S.A.

Koren, S., Walenz, B. P., Berlin, K., Miller, J. R., Bergman, N. H., and Phillippy, A. M. 2017. Canu: Scalable and accurate long-read assembly via adaptive $k$-mer weighting and repeat separation. Genome Res. 27: 722-736.

Krzywinski, M., Schein, J., Birol, I., Connors, J., Gascoyne, R., Horsman, D., Jones, S. J., and Marra, M. A. 2009. Circos: An information aesthetic for comparative genomics. Genome Res. 19:1639-1645.

Ma, L.-J., van der Does, H. C., Borkovich, K. A., Coleman, J. J., Daboussi, M.-J., Di Pietro, A., Dufresne, M., Freitag, M., Grabherr, M., Henrissat, B., Houterman, P. M., Kang, S., Shim, W.-B., Woloshuk, C., Xie, X., Xu, J.-R., Antoniw, J., Baker, S. E., Bluhm, B. H., Breakspear, A., Brown, D. W., Butchko, R. A. E., Chapman, S., Coulson, R., Coutinho, P. M., Danchin, E. G. J., Diener, A., Gale, L. R., Gardiner, D. M., Goff, S., Hammond-Kosack, K. E., Hilburn, K., Hua-Van, A., Jonkers, W., Kazan, K., Kodira, C. D., Koehrsen, M., Kumar, L., Lee, Y.-H., Li, L., Manners, J. M., Miranda-Saavedra, D., Mukherjee, M., Park, G., Park, J., Park, S.-Y., Proctor, R. H., Regev, A., Ruiz-Roldan, M. C., Sain, D., Sakthikumar, S., Sykes, S., Schwartz, D. C., Turgeon, B. G., Wapinski, I., Yoder, O., Young, S., Zeng, Q., Zhou, S., Galagan, J., Cuomo, C. A., Kistler, H. C., and Rep, M. 2010. Comparative genomics reveals mobile pathogenicity chromosomes in Fusarium. Nature 464:367-373.

Michielse, C. B., and Rep, M. 2009. Pathogen profile update: Fusarium oxysporum. Mol. Plant Pathol. 10:311-324.
Olivain, C., Trouvelot, S., Binet, M. N., Cordier, C., Pugin, A., and Alabouvette, C. 2003. Colonization of flax roots and early physiological responses of flax cells inoculated with pathogenic and nonpathogenic strains of Fusarium oxysporum. Appl. Environ. Microbiol. 69:54535462.

Rozhmina, T. A. 2002. Identification of genes for resistance to Fusarium wilt in flax. Breeding, seed production, agrotechnology, economics and primary processing of flax. [in Russian]. Torzhok 30:48-52

Rozhmina, T. A., and Loshakova, N.I. 2016. Samples of spinning and oil flax (Linum usitatissimum L.) - Sources of effective genes for resistance to Fusarium wilt and its dependence on temperature. Agricultural Biology 51:310-317 [in Russian]

Schmidt, S. M., Houterman, P. M., Schreiver, I., Ma, L., Amyotte, S., Chellappan, B., Boeren, S., Takken, F. L. W., and Rep, M. 2013. MITEs in the promoters of effector genes allow prediction of novel virulence genes in Fusarium oxysporum. BMC Genomics 14:119.

Schmidt, S. M., Lukasiewicz, J., Farrer, R., van Dam, P., Bertoldo, C., and Rep, M. 2016. Comparative genomics of Fusarium oxysporum f. sp. melonis reveals the secreted protein recognized by the Fom-2 resistance gene in melon. New Phytol. 209:307-318.

Seo, S., Pokhrel, A., and Coleman, J. J. 2020. The genome sequence of five genotypes of Fusarium oxysporum f. sp. vasinfectum: A resource for studies on Fusarium wilt of cotton. Mol. Plant-Microbe Interact 33: 138-140.

Seppey, M., Manni, M., and Zdobnov, E. M. 2019. BUSCO: Assessing genome assembly and annotation completeness. Methods Mol. Biol. 1962:227-245.

Simpson, J. T., Wong, K., Jackman, S. D., Schein, J. E., Jones, S. J., and Birol, I. 2009. ABySS: A parallel assembler for short read sequence data. Genome Res. 19:1117-1123.

Walker, B. J., Abeel, T., Shea, T., Priest, M., Abouelliel, A., Sakthikumar, S., Cuomo, C. A., Zeng, Q., Wortman, J., Young, S. K., and Earl, A. M. 2014. Pilon: An integrated tool for comprehensive microbial variant detection and genome assembly improvement. PLoS One 9:e112963.

Williams, A. H., Sharma, M., Thatcher, L. F., Azam, S., Hane, J. K., Sperschneider, J., Kidd, B. N., Anderson, J. P., Ghosh, R., Garg, G., Lichtenzveig, J., Kistler, H. C., Shea, T., Young, S., Buck, S. A. G. Kamphuis, L. G., Saxena, R., Pande, S., Ma, L. J., Varshney, R. K., and Singh, K. B. 2016. Comparative genomics and prediction of conditionally dispensable sequences in legume-infecting Fusarium oxysporum formae speciales facilitates identification of candidate effectors. BMC Genomics 17:191.

Zhang, Y., and Ma, L. J. 2017. Deciphering pathogenicity of Fusarium oxysporum from a phylogenomics perspective. Adv. Genet. 100:179-209. 